\title{
Rice Sheath Blight Disease Resistance Identified in Oryza spp. Accessions
}

\author{
B. Prasad, University of Arkansas, Rice Research and Extension Center, Stuttgart 72160; and G. C. Eizenga, \\ United States Department of Agriculture-Agricultural Research Service, Dale Bumpers National Rice Research Cen- \\ ter, Stuttgart, AR 72160
}

\begin{abstract}
Prasad, B., and Eizenga, G. C. 2008. Rice sheath blight disease resistance identified in Oryza spp. accessions. Plant Dis. 92:1503-1509.

Oryza spp., wild relatives of cultivated rice (Oryza sativa), may contain novel resistance genes for sheath blight, caused by Rhizoctonia solani, that could be used to enhance resistance to this important disease in commercial rice. To identify resistant sources for sheath blight disease, 73 Oryza genotypes were evaluated with three different methods conducted in the greenhouse, growth chamber, or laboratory because there are significant limitations to screening wild Oryza spp. under field conditions. For the microchamber method, 4-week-old seedlings were inoculated with a potato dextrose agar plug containing mycelia, covered with a 2-liter soft drink bottle, and rated 1 week after inoculation. A detached-leaf method involved placing a potato dextrose agar plug containing mycelia on the abaxial surface of a leaf section that was cut from a 5week-old plant and placed on moist filter paper in a petri dish under constant light, then evaluated after $72 \mathrm{~h}$. For the toothpick inoculation method, toothpicks colonized with mycelia were placed in the leaf collar region of plants at the panicle initiation stage, plants were placed in a growth chamber, and disease symptoms were evaluated after 7 days. The microchamber method gave a more uniform, reproducible response, and was easier to use under greenhouse conditions. Seven Oryza spp. accessions were identified as moderately resistant with three accessions classified as $O$. nivara (IRGC104705, IRGC100898, and IRGC104443) and one each as $O$. barthii (IRGC100223), O. meridionalis (IRGC105306), O. nivara/O. sativa (IRGC100943), and $O$. officinalis (IRGC105979).
\end{abstract}

Rice sheath blight disease, caused by a soilborne necrotrophic fungus Rhizoctonia solani Kühn, is one of the most important diseases of cultivated rice (Oryza sativa L.). The disease was first reported in Japan in 1910 and subsequently reported to be widespread (24). Presently, rice sheath blight is widely distributed in most rice-growing areas, including temperate, tropical, and subtropical regions, and in diverse rice production systems (17). The importance of sheath blight disease in U.S. rice cultivation was negligible until the 1970s, when more intensive crop rotation of rice with soybean (Glycine max L.) began (17). Aerial blight of soybean, also caused by $R$. solani,

Corresponding author: G. C. Eizenga

E-mail: georgia.eizenga@ars.usda.gov

This work was supported in part by the United States Department of Agriculture Cooperative State Research, Education, and Extension ServiceNational Research Initiative-Applied Plant Genomics Program entitled "RiceCAP: A coordinated research, education, and extension project for the application of genomic discoveries to improve rice in the United States" (USDA/CSREES grant 200435317-14867).

Accepted for publication 7 July 2008.

doi:10.1094/PDIS-92-11-1503

This article is in the public domain and not copyrightable. It may be freely reprinted with customary crediting of the source. The American Phytopathological Society, 2008. likely contributed to the increase in rice sheath blight (17). Most importantly, the widespread cultivation of semidwarf, high-yielding rice cultivars fertilized with high rates of nitrogen fertilizer, elevated the incidence and spread of sheath blight in the United States, especially in southern rice-growing areas (17). At the late tillering or early internode elongation growth stage, a disease lesion often is observed in the leaf sheath near the water line from germinating sclerotia, the primary source of inoculum in the field (20). Disease lesions may coalesce to form bigger lesions and the disease can spread to adjacent plants in the field. Rice sheath blight disease has been reported to cause approximately $50 \%$ yield reduction in test plots of susceptible cultivars $(17,24,25)$.

Wild relatives of rice (Oryza spp.) are a valuable source of important agronomic traits as well as genes for biotic and abiotic stress tolerance, and have been used in rice improvement programs worldwide $(2,3$, $10,13)$. The rice grassy stunt virus resistance gene from $O$. nivara Sharma et Shastry was the first report of identifying and utilizing genes from rice wild relatives in rice improvement programs (14). Resistance to bacterial blight (Xanthomonas oryzae pv. oryzae Ishiyama Dye), brown planthopper (Nilaparvata lugens Stål), white-backed planthopper (Sogatella furcifera Horváth), yellow stem borer (Scirpophaga incertulas Walker), rice tungro disease (both rice tungro spherical and bacilliform viruses), and stem rot caused by Sclerotium oryzae Catt. have been identified in wild Oryza spp., and some of these resistance genes have been transferred to cultivated rice $(3,19)$. Complete field resistance to the rice sheath blight pathogen has not been identified, but partial resistance has been reported $(1,21)$. Sheath blight resistance genes were identified in O. minuta J. S. Presl. ex C. B. Presl. (IRGC101089) and O. rufipogon Griff. (IRGC100907) accessions (1). Rice sheath blight resistance genes from $O$. officinalis Wall ex Watt were transferred into cultivated rice through the backcross breeding approach (16). These results suggest that rice wild relatives possess important sources of resistance for sheath blight disease and could be useful in developing resistant cultivars or germplasm in an adapted genetic background.

Various screening methods have been tested to identify sources of resistance to sheath blight in rice germplasm. Of these methods, field screening at the reproductive stage is the most widely used $(7,23)$, but this method requires grams of seed and the plants to be near maturity, and is sensitive to environmental factors (23). Temperature, humidity, maturity date, and plant height are all important factors which contribute to sheath blight disease variation under field conditions. Recently, Park et al. (22) described three inoculation methods (agar block, liquid cultured mycelia ball, and mycelia suspension) under greenhouse conditions and found that liquid cultured mycelia ball was the best method for successful inoculation. Most wild Oryza spp. have a seed-shattering characteristic and are treated as weeds; thus, screening these accessions for sheath blight resistance in the field is not recommended. Moreover, wild Oryza spp. do not possess morphological features like a moderate plant height or closed canopy that favor disease development under field conditions. To circumvent these problems, Eizenga et al. (6) described a growth chamber screening method for sheath blight on Oryza spp. using the toothpick inoculation technique which requires the plants to grow until the panicle initiation stage when toothpicks colonized with $R$. solani mycelia are placed in the leaf collar region. For the detached-leaf method, cut leaf pieces from 4-week-old rice plants were put in a petri dish and a block of potato dextrose agar (PDA) containing 2-day-old mycelia of $R$. solani was placed on the leaf surface (12). 
Table 1. Mean sheath blight visual ratings and disease measurements obtained for the Oryza spp. accessions, introgression lines and check cultivars evaluated using microchamber and detached-leaf method

\begin{tabular}{|c|c|c|c|c|c|}
\hline \multirow[b]{2}{*}{ Genotypes $^{\mathrm{a}}$} & \multirow[b]{2}{*}{ IRGC, PI no.b } & \multicolumn{2}{|c|}{ Microchamber method } & \multicolumn{2}{|c|}{ Detached-leaf method } \\
\hline & & Disease index ${ }^{c}$ & Visual ratingd & Lesion length ${ }^{e}$ & Visual rating $f$ \\
\hline Oryza nivara/O. sativa & IRGC100943 & 3.4 & 3.7 & 5.8 & 4.0 \\
\hline O. meridionalis & IRGC105306 & 3.6 & 5.0 & 5.4 & 5.0 \\
\hline O. meridionalis & IRGC103317 & 3.8 & 4.3 & 5.7 & 6.0 \\
\hline O. barthii & IRGC100223 & 4.2 & 5.0 & 4.4 & 4.0 \\
\hline O. nivara & IRGC100898 & 4.2 & 5.0 & 6.2 & 4.0 \\
\hline O. nivara & IRGC105316 & 4.2 & 4.3 & 6.8 & 6.0 \\
\hline O. officinalis & IRGC105979 & 4.3 & 4.3 & 5.0 & 4.0 \\
\hline O. meridionalis & IRGC105300 & 4.3 & 4.3 & 6.6 & 6.0 \\
\hline O. barthii & IRGC100936 & 4.3 & 4.3 & 6.7 & 6.0 \\
\hline O. glaberrima & IRGC96717 & 4.3 & 3.7 & 8.6 & 7.0 \\
\hline O. nivara & IRGC104443 & 4.4 & 5.0 & 5.2 & 4.0 \\
\hline O. nivara & IRGC104705 & 4.4 & 5.0 & 6.1 & 4.0 \\
\hline O. glaberrima & PI232865 & 4.4 & 4.0 & 8.3 & 6.0 \\
\hline$O$. nivara/O. sativa & IRGC100606 & 4.4 & 5.0 & 11.6 & 7.0 \\
\hline O. barthii & IRGC104304 & 4.5 & 5.0 & 7.4 & 6.0 \\
\hline O. glumaepatula & IRGC105670 & 4.6 & 5.0 & 9.6 & 6.0 \\
\hline O. barthii & IRGC100935 & 4.7 & 6.0 & 6.9 & 5.0 \\
\hline O. nivara & PI590407 & 4.9 & 5.0 & 12.6 & 7.0 \\
\hline O. nivara & IRGC100593 & 5.0 & 5.3 & 7.8 & 6.0 \\
\hline O. latifolia & IRGC100967 & 5.0 & 5.0 & 10.1 & 7.0 \\
\hline O. barthii & IRGC105609 & 5.1 & 5.0 & 8.6 & 8.0 \\
\hline IR71033-4-1-12 & - & 5.2 & 4.3 & 8.3 & 5.0 \\
\hline O. barthii & PI590399 & 5.2 & 5.0 & 9.3 & 7.0 \\
\hline O. rufipogon & PI590424 & 5.4 & 5.0 & 7.7 & 7.0 \\
\hline O. nivara & PI590426 & 5.4 & 5.0 & 8.1 & 7.0 \\
\hline O. alta & PI590398 & 5.4 & 5.0 & 9.1 & 7.0 \\
\hline O. rufipogon/O. nivara & IRGC100907 & 5.5 & 5.7 & 5.9 & 5.0 \\
\hline O. barthii & IRGC104286 & 5.5 & 7.0 & 9.0 & 8.0 \\
\hline O. nivara & IRGC101524 & 5.6 & 7.0 & 9.6 & 6.0 \\
\hline Oryza sp. & PI346369 & 5.6 & 5.7 & 9.1 & 9.0 \\
\hline O. latifolia & IRGC100169 & 5.7 & 7.0 & 10.7 & 8.0 \\
\hline O. glumaepatula & IRGC105465 & 5.8 & 5.0 & 10.0 & 6.0 \\
\hline O. glaberrima & PI590403 & 5.8 & 5.3 & 10.1 & 7.0 \\
\hline O. barthii & PI236393 & 5.8 & 6.3 & 9.1 & 8.0 \\
\hline o. nivara & IRGC102169 & 5.9 & 7.0 & 11.1 & 6.0 \\
\hline O. officinalis & IRGC101399 & 5.9 & 5.0 & 11.3 & 8.0 \\
\hline IR31917-45-3-2-3 & - & 6.0 & 5.0 & 6.1 & 5.0 \\
\hline O. rufipogon & PI590423 & 6.1 & 5.0 & 5.3 & 4.0 \\
\hline o. nivara & PI360790 & 6.1 & 5.7 & 11.7 & 9.0 \\
\hline O. rufipogon & PI590420 & 6.1 & 9.0 & 13.5 & 9.0 \\
\hline O. nivara & PI590410 & 6.2 & 6.3 & 9.0 & 6.0 \\
\hline O. nivara & PI590409 & 6.2 & 7.7 & 13.7 & 7.3 \\
\hline IR71033-4-1-10 & - & 6.3 & 5.7 & 6.6 & 5.0 \\
\hline O. australiensis & IRGC103318 & 6.3 & 7.0 & 8.7 & 5.0 \\
\hline IR71033-4-1-4 & - & 6.3 & 5.7 & 7.7 & 6.0 \\
\hline O. nivara & PI590425 & 6.3 & 5.3 & 8.2 & 7.0 \\
\hline O. rufipogon & IRGC105491 & 6.3 & 6.3 & 8.5 & 7.0 \\
\hline $87-Y-550$ & PI566666 & 6.4 & 5.7 & 5.7 & 5.0 \\
\hline O. rufipogon & PI590419 & 6.5 & 5.0 & 5.0 & 5.0 \\
\hline IR31917-45-3-2-2 & - & 6.5 & 7.0 & 6.2 & 6.0 \\
\hline O. rufipogon & PI590422 & 6.5 & 5.0 & 6.7 & 6.0 \\
\hline O. nivara/O. sativa & IRGC100200 & 6.6 & 6.3 & 9.4 & 7.0 \\
\hline O. barthii & PI237987 & 6.6 & 6.3 & 10.4 & 9.0 \\
\hline O. latifolia & IRGC103808 & 6.7 & 7.7 & 14.0 & 9.0 \\
\hline O. nivara & IRGC100195 & 6.8 & 5.7 & 11.9 & 9.0 \\
\hline O. nivara & PI360791 & 6.9 & 6.0 & 6.7 & 5.0 \\
\hline Oryza sp. & PI590414 & 6.9 & 7.3 & 6.8 & 5.0 \\
\hline IR71033-4-1-5 & - & 7.0 & 5.7 & 6.8 & 5.0 \\
\hline O. alta & PI590397 & 7.0 & 6.3 & 11.8 & 7.0 \\
\hline O. officinalis & PI590412 & 7.1 & 6.3 & 6.1 & 5.0 \\
\hline O. nivara & IRGC100897 & 7.1 & 7.0 & 10.4 & 9.0 \\
\hline Oryza sp. & PI346360 & 7.1 & 7.7 & 10.4 & 9.0 \\
\hline Oryza sp. & PI346362 & 7.2 & 7.7 & 10.1 & 9.0 \\
\hline
\end{tabular}

a Oryza spp. accessions in bold were evaluated with toothpick method (Table 3). LSD = least significant difference.

${ }^{\mathrm{b}}$ Accessions missing a plant introduction (PI) or International Rice Germplasm Collection (IRGC) number are blast-resistant $O$. minuta introgression lines (IR71033-4-1) and their O. sativa parental lines (IR31917-45-3-2) obtained from the International Rice Research Institute, Philippines.

${ }^{c}$ Disease index was calculated based on the formula (lesion length/culm length) $\times 9$, where lesion length was measured as the length of lesion along the length of culm after Jia et al. (11).

d Visual rating was based on 0 -to- 9 scale, where $0=$ no lesion and $9=$ entire culm has a lesion.

${ }^{\mathrm{e}}$ Lesion length $(\mathrm{cm})$ was measured as the total lesion length from the point of inoculation upward and downward on the cut leaf surface.

${ }^{f}$ Visual rating was based on the extent of the lesion in the cut leaf, where $0=$ no lesion and $9=$ entire leaf segment has lesion. 


\begin{tabular}{|c|c|c|c|c|c|}
\hline \multirow[b]{2}{*}{ Genotypes $^{\mathrm{a}}$} & \multirow[b]{2}{*}{ IRGC, PI no. ${ }^{b}$} & \multicolumn{2}{|c|}{ Microchamber method } & \multicolumn{2}{|c|}{ Detached-leaf method } \\
\hline & & Disease index ${ }^{c}$ & Visual rating $^{d}$ & Lesion length $^{\mathrm{e}}$ & Visual rating ${ }^{\mathrm{f}}$ \\
\hline O. nivara/O. sativa & IRGC100917 & 7.5 & 7.7 & 7.0 & 6.0 \\
\hline O. officinalis & IRGC101121 & 7.7 & 7.7 & 8.7 & 7.0 \\
\hline Oryza sp. & PI590415 & 8.1 & 9.0 & 11.9 & 9.0 \\
\hline Oryza sp. & PI346356 & 8.4 & 9.0 & 10.8 & 8.0 \\
\hline 'Cocodrie' & PI606331 & 6.7 & 7.0 & 8.2 & 6.0 \\
\hline 'Lemont' & PI475833 & 7.0 & 8.0 & 8.7 & 7.0 \\
\hline 'Ahrent' & PI628972 & 5.1 & 5.7 & 8.2 & 6.0 \\
\hline 'Bengal' & PI561735 & 5.5 & 5.0 & 7.2 & 5.0 \\
\hline 'Jasmine 85 ' & PI595927 & 4.1 & 4.0 & 5.5 & 4.0 \\
\hline ‘TeQing' & PI536047 & 3.9 & 3.3 & 5.4 & 4.0 \\
\hline Mean & $\ldots$ & 5.7 & 5.8 & 8.4 & 6.4 \\
\hline $\operatorname{LSD}(0.05)$ & $\ldots$ & 1.0 & 1.2 & 1.7 & 0.8 \\
\hline
\end{tabular}

Recently, a microchamber technique was reported (11).

The objectives of this study were to (i) evaluate several sheath blight disease screening methods on wild Oryza spp. germplasm and (ii) identify possible resistance sources from the wild Oryza spp. accessions.

\section{MATERIALS AND METHODS}

Included in this study were 60 wild Oryza spp. accessions representing 15 different species; one stem-rot-resistant introgression line; four rice-blast-resistant introgression lines, including the two $O$. sativa parental lines obtained from the International Rice Research Institute (IRRI); and six $O$. sativa reference cultivars (Table 1). Accessions with a plant introduction (PI) number are available from the United States Department of Agriculture-Agricultural Research Service National Plant Germplasm System (http://www.ars-grin.gov/npgs) and those with an IRGC number from IRRI, Philippines (http://www.irri.org). The reference cultivars were previously scored as moderately resistant to highly susceptible for sheath blight disease resistance under both greenhouse and field conditions $(6,11)$.

Microchamber method. The inoculum preparation was according to Jia et al. (11) using a wild-type, slow-growing isolate of $R$. solani (RR0140-1). Previously, this isolate produced consistent symptoms on reference cultivars and was confirmed as a true isolate of $R$. solani by molecular data (27). Dehulled seed were incubated on a Knudson modified orchid medium (SigmaAldrich Inc., St. Louis) with agar at 10.5 $\mathrm{g} /$ liter in an incubator set at $30^{\circ} \mathrm{C}, 12 \mathrm{~h}$ of light, and $12 \mathrm{~h}$ of darkness for uniform germination. Seven-day-old seedlings were transplanted in a mixture of one part Redi-Earth (Sun Gro Horticulture, Bellevue, WA) potting soil to two parts steamsterilized soil in order to eliminate any effect of preexisting soilborne $R$. solani inoculum. Four-week-old seedlings were inoculated with a PDA plug containing $R$. solani mycelia. Subsequently, the plants were covered with a microchamber adapted from a 2-liter soft drink bottle with the base and lid removed. Humidity inside the chamber measured $>85 \%$ and temperature ranged from 30 to $35^{\circ} \mathrm{C}$. Inoculated plants were maintained on the greenhouse bench for 7 to 10 days. Each pot represented a replication and contained three inoculated plants. The experiment was conducted in a completely randomized design with three replications.

Plants were evaluated for disease response 7 to 10 days after inoculation. A disease index ranging from 0 to 9 also was determined as follows: each seedling was cut from the base, and the lesion length along the base of the culm and total culm length were measured. The disease index was calculated as lesion length divided by culm length and multiplied by 9 as previously described (11). Disease index $<4$ was considered to be moderately resistant, 5 to 6 moderately susceptible, and 7 to 9 highly susceptible. A visual rating was made for each seedling based on a 0 -to- 9 scale, where 0 indicated no lesion was observed and 9 indicated that 90 to $100 \%$ of the culm area, including the leaf, had lesions. Visual scores of 1 to 8 represented 10 to $80 \%$ of diseased plant area.

Detached-leaf method. Inoculum preparation and test entries for the detached-leaf inoculation method were similar to the microchamber method described above. The inoculation method was according to Jia et al. (12). Plants were grown in the greenhouse until the V5 stage (4). The second youngest leaf from the main tiller was cut into a 16-cm-long piece, placed on a moistened filter paper, and kept in a 24-by-24-by-1.8-cm petri dish. For each entry, four leaves were used as one replicate with four replications per entry in a completely randomized design. Colonized PDA blocks, $7 \mathrm{~mm}$ in diameter, were excised with a 1-ml inverted disposable pipette tip from the PDA plate and placed on the abaxial surface of each leaf piece. The leaves were incubated under ambient laboratory conditions with constant cool fluorescent white light for $72 \mathrm{~h}$ at $25^{\circ} \mathrm{C}$. The filter paper was kept moist with sterile water. After $72 \mathrm{~h}$, the lesion length of each cut leaf piece was measured and each leaf was visually rated using a 0 to- 9 scale with 0 having no lesion to 9 having 90 to $100 \%$ of the leaf surface covered with lesions. Visual scores of 1 to 8 represented 10 to $80 \%$ diseased leaf area.

Toothpick method. Previously, 19 wild Oryza spp. accessions were screened for sheath blight resistance using a toothpick inoculation assay (6). In the current study, a subset of 38 accessions was screened using primary or secondary tillers using this method (6). The experiment was conducted as a completely randomized design using three plants per pot as a replication with three replications. The data were averaged from five tillers of each plant. Toothpicks colonized with mycelium were placed at the leaf collar region and inoculated plants were covered with a transparent plastic bag and kept in a growth chamber set at $14 \mathrm{~h}$ of light, $10 \mathrm{~h}$ of darkness, $100 \%$ relative humidity, and $28^{\circ} \mathrm{C}$ (6). After 7 days, each plant was rated visually using a 0 -to- 9 scale, where $0=$ no lesion and $9=$ lesions covering the whole plant, including the panicles. Visual scores of 1 to 8 signified that 10 to $80 \%$ of the plant area had disease lesions. Leaves were numbered from 1 to 3 , where 1 was the oldest leaf, closest to the soil. The total lesion length was determined by adding the lesion length from the point of inoculation at the leaf collar, up the leaf blade, and down the leaf sheath. The relative lesion length of each leaf was calculated by dividing the total leaf length into the total lesion length, expressed as a percentage. Combinations of lesion length from the three leaves (leaves $1+2$, leaves $1+3$, leaves $2+3$, and leaves $1+2+3$ ) also were calculated.

Data analysis. Data collected from the three methods were analyzed using the General Linear Models procedure (PROC GLM) in the SAS system (SAS, Cary, NC). Correlation coefficients between the components of each individual method and between different methods were determined by the correlation procedure (PROC CORR) in SAS. Least significance differ- 
Table 2. Pearson's correlation coefficients between the three sheath blight screening methods for 73 genotypes using microchamber and detached-leaf method (Table 1) and 38 accessions using toothpick method (Table 3) ${ }^{\mathrm{a}}$

\begin{tabular}{|c|c|c|c|c|c|}
\hline & $\begin{array}{l}\text { Microchamber } \\
\text { (visual score) }\end{array}$ & $\begin{array}{l}\text { Microchamber } \\
\text { (disease index) }\end{array}$ & $\begin{array}{l}\text { Detached-leaf } \\
\text { (visual score) }\end{array}$ & $\begin{array}{l}\text { Detached-leaf } \\
\text { (lesion length) }\end{array}$ & $\begin{array}{c}\text { Toothpick } \\
\text { (visual score) }\end{array}$ \\
\hline Microchamber (visual score) & $\ldots$ & $\ldots$ & $\ldots$ & $\ldots$ & $\ldots$ \\
\hline Microchamber (disease index) & $0.79 * *$ & $\ldots$ & $\ldots$ & $\ldots$ & $\ldots$ \\
\hline Detached-leaf (visual score) & $0.51 * *$ & $0.45 * *$ & $\ldots$ & $\ldots$ & $\ldots$ \\
\hline Detached-leaf (lesion length) & $0.52 * *$ & $0.39 * *$ & $0.81 * *$ & $\ldots$ & $\ldots$ \\
\hline Toothpick (visual score) & $0.32 *$ & 0.08 & 0.08 & 0.20 & $\ldots$ \\
\hline Toothpick (lesion length) ${ }^{\mathrm{b}}$ & $0.35 *$ & 0.21 & 0.20 & 0.28 & $0.78 * *$ \\
\hline
\end{tabular}

a Asterisks: $*$ and $* *=P<0.05$ and 0.01 , respectively.

${ }^{\mathrm{b}}$ Combined lesion length from second and third leaf.

Table 3. Mean sheath blight visual ratings and disease lesion measurements for the Oryza spp. accessions evaluated using the toothpick method of inoculation

\begin{tabular}{|c|c|c|c|c|c|c|c|c|c|c|c|c|}
\hline \multirow[b]{3}{*}{ Genotypes $^{d}$} & \multirow[b]{3}{*}{ IRGC, PI no.e } & \multirow[b]{3}{*}{$\mathbf{V R} \mathbf{R}^{\mathbf{f}}$} & \multicolumn{10}{|c|}{ Leaves 1, 2, 3, and combinations } \\
\hline & & & \multicolumn{3}{|c|}{ Lesion lengtha } & \multicolumn{3}{|c|}{ Relative lesion length ${ }^{b}$} & \multicolumn{4}{|c|}{ Combined lesion length ${ }^{c}$} \\
\hline & & & 1 & 2 & 3 & $\mathbf{1}$ & 2 & 3 & $1+2$ & $1+3$ & $2+3$ & $1+2+3$ \\
\hline Oryza barthii & PI590399 & 4.0 & 5.0 & 6.0 & 5.0 & 6.5 & 9.0 & 8.0 & 11.0 & 10.0 & 11.0 & 16.0 \\
\hline O. nivara/O. sativa & IRGC100917 & 4.5 & 8.5 & 6.0 & 4.0 & 19.0 & 11.0 & 9.0 & 14.5 & 12.5 & 10.0 & 18.5 \\
\hline O. officinalis & IRGC101121 & 4.5 & 5.0 & 5.0 & 5.0 & 19.5 & 15.5 & 15.5 & 10.0 & 10.0 & 10.0 & 15.0 \\
\hline Oryza sp. & PI346369 & 4.5 & 4.0 & 6.0 & 4.0 & 7.0 & 13.0 & 8.0 & 10.0 & 8.0 & 10.0 & 14.0 \\
\hline O. alta & PI590398 & 4.5 & 9.0 & 7.0 & 4.0 & 16.5 & 11.5 & 6.0 & 16.0 & 13.0 & 11.0 & 20.0 \\
\hline O. nivara & PI590425 & 4.5 & 13.0 & 9.0 & 5.5 & 21.5 & 17.0 & 11.0 & 22.0 & 18.5 & 14.0 & 27.0 \\
\hline O. nivara & PI590426 & 4.5 & 8.0 & 8.0 & 8.0 & 19.5 & 17.0 & 17.0 & 16.0 & 16.0 & 16.0 & 24.0 \\
\hline O. rufipogon & PI590424 & 4.5 & 56.0 & 12.5 & 8.5 & 86.0 & 19.0 & 15.5 & 68.0 & 64.0 & 20.5 & 76.5 \\
\hline O. alta & PI590397 & 5.0 & 7.5 & 5.0 & 6.0 & 13.0 & 6.5 & 9.0 & 12.5 & 13.5 & 11.0 & 18.5 \\
\hline O. rufipogon & PI590419 & 5.0 & 58.0 & 13.0 & 8.0 & 86.0 & 22.5 & 15.0 & 71.0 & 65.5 & 21.0 & 78.5 \\
\hline O. rufipogon & PI590423 & 5.0 & 17.5 & 15.0 & 7.5 & 34.0 & 22.0 & 14.5 & 32.5 & 25.0 & 22.5 & 40.0 \\
\hline Oryza sp. & PI590414 & 5.0 & 20.0 & 12.5 & 12.5 & 61.0 & 41.0 & 41.0 & 32.5 & 32.5 & 24.5 & 44.5 \\
\hline O. latifolia & IRGC103808 & 5.0 & 12.5 & 12.0 & 13.0 & 24.0 & 20.0 & 30.5 & 24.5 & 25.5 & 25.0 & 37.5 \\
\hline Oryza sp. & PI346360 & 5.0 & 18.5 & 16.0 & 12.0 & 37.0 & 30.5 & 19.0 & 34.0 & 30.0 & 27.5 & 46.0 \\
\hline O. glaberrima & PI590403 & 5.0 & 44.5 & 18.0 & 14.5 & 67.0 & 30.5 & 25.5 & 62.5 & 59.0 & 32.5 & 77.0 \\
\hline O. nivara & PI590407 & 5.5 & 41.0 & 7.0 & 10.0 & 56.0 & 10.5 & 18.0 & 48.0 & 51.0 & 17.0 & 58.0 \\
\hline O. nivara & IRGC102169 & 5.5 & 9.0 & 8.0 & 10.0 & 28.0 & 22.0 & 34.0 & 17.0 & 19.0 & 18.0 & 27.0 \\
\hline O. rufipogon & PI590422 & 5.5 & 30.0 & 10.0 & 8.5 & 60.0 & 15.5 & 15.5 & 40.0 & 38.5 & 18.5 & 48.5 \\
\hline O. nivara & PI360791 & 5.5 & 6.0 & 9.0 & 11.0 & 9.0 & 13.0 & 18.0 & 15.0 & 16.5 & 20.0 & 25.5 \\
\hline O. glaberrima & IRGC96717 & 5.5 & 17.0 & 10.0 & 12.0 & 37.0 & 25.0 & 30.0 & 27.0 & 29.0 & 22.0 & 39.0 \\
\hline O. barthii & IRGC104304 & 5.5 & 19.5 & 15.0 & 12.0 & 42.5 & 25.0 & 22.5 & 34.0 & 31.0 & 26.5 & 46.0 \\
\hline Oryza sp. & PI346362 & 5.5 & 23.5 & 18.0 & 9.0 & 50.0 & 40.5 & 20.0 & 41.5 & 32.5 & 27.0 & 50.5 \\
\hline O. rufipogon/O. nivara & IRGC100907 & 5.5 & 30.0 & 16.5 & 11.5 & 60.5 & 31.0 & 22.5 & 46.5 & 41.5 & 28.0 & 58.0 \\
\hline O. nivara & PI360790 & 5.5 & 14.0 & 14.0 & 14.0 & 37.0 & 37.0 & 37.0 & 28.5 & 28.5 & 28.5 & 42.0 \\
\hline Oryza sp. & PI346356 & 5.5 & 13.0 & 15.5 & 15.5 & 22.5 & 32.0 & 32.0 & 28.0 & 28.0 & 30.5 & 43.0 \\
\hline O. barthii & IRGC100936 & 5.5 & 19.5 & 16.0 & 15.5 & 41.5 & 31.5 & 26.5 & 35.0 & 34.5 & 31.0 & 50.5 \\
\hline O. nivara & IRGC104443 & 5.5 & 20.0 & 14.5 & 19.0 & 45.0 & 27.5 & 34.0 & 34.0 & 38.5 & 33.0 & 53.0 \\
\hline O. officinalis & PI590412 & 6.0 & 42.5 & 14.5 & 13.0 & 97.5 & 30.0 & 26.0 & 57.0 & 55.5 & 27.5 & 70.0 \\
\hline O. barthii & IRGC100223 & 6.0 & 19.0 & 16.5 & 12.5 & 40.5 & 31.5 & 45.5 & 35.5 & 31.5 & 29.0 & 48.0 \\
\hline O. nivara & PI590410 & 6.0 & 11.0 & 16.0 & 16.0 & 38.0 & 48.0 & 48.0 & 27.0 & 27.0 & 32.0 & 43.0 \\
\hline O. officinalis & IRGC105979 & 6.5 & 22.0 & 15.5 & 10.0 & 51.0 & 31.0 & 19.0 & 37.5 & 32.0 & 25.5 & 47.5 \\
\hline O. nivara & PI590409 & 6.5 & 34.0 & 24.0 & 9.5 & 75.5 & 51.0 & 21.0 & 58.0 & 43.5 & 34.0 & 68.0 \\
\hline Oryza sp. & PI590415 & 6.5 & 19.0 & 16.0 & 18.0 & 39.5 & 40.5 & 54.5 & 35.0 & 37.0 & 34.0 & 53.0 \\
\hline O. barthii & PI236393 & 6.5 & 23.0 & 15.5 & 20.5 & 53.0 & 27.0 & 36.0 & 38.5 & 43.5 & 36.0 & 59.0 \\
\hline O. barthii & PI237987 & 6.5 & 22.0 & 18.5 & 18.0 & 54.5 & 41.0 & 33.0 & 40.5 & 40.0 & 36.5 & 58.5 \\
\hline O. glaberrima & PI232865 & 6.5 & 45.0 & 31.0 & 34.0 & 65.5 & 43.5 & 47.5 & 76.0 & 79.0 & 65.0 & 110.0 \\
\hline O. latifolia & IRGC100967 & 7.0 & 41.0 & 21.0 & 24.5 & 82.0 & 32.5 & 33.5 & 62.0 & 65.5 & 45.5 & 86.5 \\
\hline O. nivara & IRGC101524 & 7.5 & 48.5 & 42.5 & 28.5 & 100.0 & 95.5 & 61.5 & 90.5 & 77.0 & 71.0 & 119.0 \\
\hline 'Cocodrie' & PI606331 & 6.5 & 19.0 & 18.5 & 15.5 & 67.0 & 49.5 & 39.0 & 37.5 & 34.5 & 34.0 & 53.0 \\
\hline 'Lemont' & PI475833 & 7.0 & 17.5 & 15.0 & 18.0 & 61.0 & 47.5 & 53.0 & 32.5 & 35.5 & 33.0 & 50.5 \\
\hline 'Ahrent' & PI628972 & 6.5 & 12.0 & 21.0 & 9.0 & 17.5 & 28.0 & 13.0 & 33.0 & 21.0 & 30.0 & 42.0 \\
\hline 'Bengal' & PI561735 & 6.0 & 8.5 & 9.0 & 17.0 & 13.0 & 14.5 & 34.0 & 17.5 & 25.5 & 26.0 & 34.5 \\
\hline 'Jasmine 85 ' & PI595927 & 3.5 & 13.5 & 13.0 & 10.0 & 26.0 & 24.0 & 18.0 & 26.5 & 23.0 & 23.0 & 36.0 \\
\hline 'TeQing' & PI536047 & 3.5 & 16.0 & 6.0 & 5.0 & 32.5 & 11.5 & 11.0 & 22.0 & 21.0 & 11.0 & 27.0 \\
\hline Mean & $\ldots$ & 5.5 & 22.0 & 14.6 & 12.5 & 44.7 & 29.6 & 24.6 & 36.5 & 18.7 & 27.1 & 49.0 \\
\hline $\mathrm{LSD}(0.05)$ & $\ldots$ & 1.2 & 10.2 & 9.5 & 6.5 & 20.1 & 10.2 & 10.5 & 15.7 & 13.3 & 12.3 & 18.3 \\
\hline
\end{tabular}

${ }^{a}$ Lesion length $(\mathrm{cm})$ was calculated for each leaf as the length of lesion from the collar down the leaf sheath and from the collar up the leaf blade.

${ }^{b}$ Relative lesion length was calculated as the proportion of leaf length that is diseased.

${ }^{c}$ Represents the lesion length $(\mathrm{cm})$ in various combinations of the first three leaves as described in the column heading, considering the first leaf as the oldest and closest to the ground.

d Only Oryza spp. accessions not previously evaluated were included in this screening (6). LSD = least significant difference.

e $\mathrm{PI}=$ plant introduction; IRGC = International Rice Germplasm Collection .

${ }^{\mathrm{f}}$ Visual rating (VR) was based on a 0 -to-9 scale, where $0=$ no lesion and $9=$ lesions covering all leaves and panicle. Visual rating was assigned on a wholeplant basis. 
ence (LSD) values were calculated using PROC GLM. Data from the microchamber and detached leaf methods were analyzed with the multivariate statistical package (MVSP) software (15) to group all 73 genotypes into clusters. The cluster is reported as an unweighted pair group method using arithmetic means and the Euclidean distance algorithm.

\section{RESULTS}

Microchamber method. The microchamber created a hot and humid environment, with temperatures ranging from 30 to $35^{\circ} \mathrm{C}$ and relative humidity $>85 \%$, resulting in rapid development of the sheath blight disease. Even though the temperature was slightly higher and the humidity slightly lower than optimum, favorable disease development was achieved and significant differences $(P<0.01)$ were observed between the tested genotypes for both disease index and visual rating. Rice reference cultivars Cocodrie and Lemont were rated as highly susceptible, Ahrent and Bengal moderately susceptible, and Jasmine 85 and TeQing moderately resistant (Table 1 ). The reaction of the Oryza spp. accessions ranged from highly susceptible to moderately resistant. Eighteen Oryza spp. accessions had a disease index less then 5.0 (Table 1). These accessions included the species $O$. barthii A. Chev., O. glaberrima Steud., O. glumaepatula Steud., O. meridionalis $\mathrm{Ng}$, O. nivara, $O$. nivara/O. sativa, and $O$. officinalis. Within the moderately resistant accessions, three had a disease index less than Jasmine 85 and three were visually rated as similar to Jasmine 85 . One $O$. nivara/O. sativa (IRGC100943) accession had a lower disease index (3.4) than the moderately resistant cvs. Jasmine 85 and TeQing. The ratings of the reference cultivars were similar to the rating based on the field screening methods reported earlier $(6,11)$. The visual rating of the 73 genotypes was highly correlated with their respective disease index ( $r=0.79, P<0.01$; Table 2).

Detached-leaf method. Moderately resistant Jasmine 85 and TeQing had the shortest lesion lengths $(5.5$ and $5.4 \mathrm{~cm}$, respectively) among the reference cultivars (Table 1). Lesion length of the accessions ranged from 4.0 to $14.0 \mathrm{~cm}$ with six accessions represented by $O$. barthii, $O$. nivara, $O$. meridionalis, $O$. officinalis, and $O$. rufipogon, having a lesion length that was equal to or less than the most resistant reference cv. Jasmine 85 (Table 1). By contrast, 31 accessions had a lesion length equal to or greater than the highly susceptible reference cv. Lemont (8.7 $\mathrm{cm})$. Visual ratings of the Oryza spp. accessions ranged from 4.0 to 9.0 , but not one of the ratings was lower than the moderately resistant checks Jasmine 85 and TeQing, even though seven accessions fell into the same categories as Jasmine 85 and TeQing (Table 1). The correlation coefficient between the visual rating and lesion length was strong ( $r=$ $0.81, P<0.01$; Table 2).
Toothpick method. Primary and secondary tillers from 38 Oryza spp. accessions and six check cultivars were screened for sheath blight resistance with the toothpick screening method. Visual ratings of the accessions ranged from 4.0 to 7.5 (Table $3)$. For the first three leaves, the lesion length was measured and relative lesion length was calculated, as well as the combined lesion length (leaves $1+2$, leaves $1+3$, leaves $2+3$, and leaves $1+2+3$ ). When different disease measurements were analyzed for their relationship with each other, the best correlation was observed between the visual rating and combined lesion length of the second and third leaves ( $r=$ $0.78, P<0.01$; Table 2). None of the 38 accessions had a visual rating less than the two moderately resistant checks, Jasmine 85 and TeQing. Among the three leaves, mean lesion length across the test entries was highest for the first leaf followed by the second and third leaves, and these trends were similar to the relative lesion lengths of the respective leaves. Six accessions (O. alta PI590397, PI590398; $O$. barthii PI590399; O. nivara/O. sativa IRGC100917; O. officinalis IRGC101121; and Oryza sp. PI346369) had equal or shorter combined lesion length for leaf 2 and 3 than the moderately resistant check TeQing (Table 3).

Correlation analysis between the three methods (Table 2) revealed highly significant correlations $(P<0.01)$ between the microchamber and detached-leaf methods

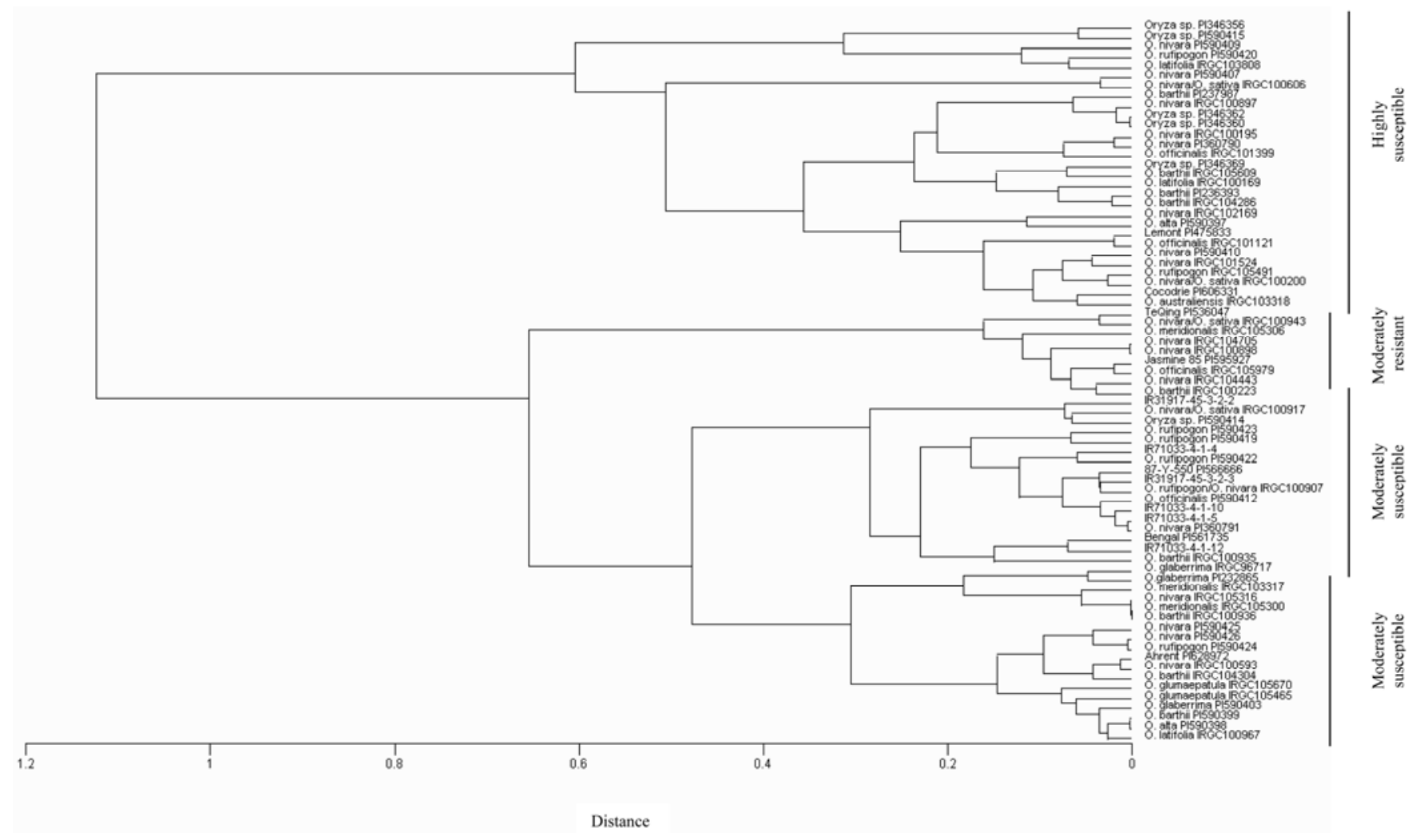

Fig. 1. Unweighted pair group method using arithmetic means cluster of the 73 Oryza spp. genotypes, constructed with a Euclidean distance algorithm (15) based on data reported in Table 1 from the microchamber and detached-leaf methods of screening for sheath blight disease. The vertical axis represents the genotypes and the horizontal axis the distance between the genotypes. 
for both the disease components for each method but low and inconsistent correlation between the toothpick method and both the microchamber and detached-leaf methods.

Cluster analysis. The 73 genotypes included in this study were grouped into clusters using a distance-based analysis of the data obtained from the microchamber and detached-leaf methods (Fig. 1). The accessions assayed with the toothpick method were excluded from the cluster analysis because data was collected for only 38 genotypes (Table 3 ) and the data collection method for the 19 genotypes previously reported varied slightly. The accessions grouped into four clusters with the largest cluster containing 27 highly susceptible accessions, including the two reference cvs. Cocodrie and Lemont, known to be highly susceptible. The second cluster contained seven moderately resistant accessions along with the known moderately resistant reference cvs. Jasmine 85 and TeQing. The accessions included in the moderately resistant cluster were three O. nivara accessions (IRGC104705, IRGC100898, and IRGC104443); O. nivara/O. sativa (IRGC100943), which is very tall; $O$. officinalis (IRGC105979), reported to be an A-genome species (8) that clustered with several $O$. barthii accessions based on microsatellite markers (5); O. meridionalis (IRGC105306); and O. barthii (IRGC100223). The other two clusters contained the moderately susceptible accessions along with the moderately susceptible reference cvs. Bengal in one cluster and Ahrent in the other cluster.

\section{DISCUSSION}

The wild Oryza spp. accessions may offer a potential source of genes for improving sheath blight resistance in the rice gene pool worldwide, especially because only limited resistance to rice sheath blight has been identified in the cultivated rice gene pool (6). For research purposes, it is better to grow the wild Oryza spp. in the greenhouse or growth chamber due to the seedshattering characteristic of most accessions; thus, it is critical that a suitable screening method not dependent on field testing be identified.

Several different sheath blight screening methods have been tested at IRRI, Philippines (20). One of these methods was a seedling method using 3-week-old seedlings inoculated with the $R$. solani fungus cultured in rice straw. The test results using the seedlings were similar to results obtained from the field evaluation of the same set of cultivars (20). Singh et al. (26) inoculated 4-week-old plants in the greenhouse with immature sclerotia, mature sclerotia, and mycelia. This study concluded that the amount of inoculum was the most important factor for uniform development of disease symptoms, and 4day-old immature sclerotia were the best in differentiating the disease response using this inoculation method.

Most recently, Jia et al. (11) reported a sheath blight screening method adapted from Bangladesh and conducted in the greenhouse utilizing a microchamber which was described as fast, accurate, repeatable, and potentially correlated with field screening results. The microchamber creates a high-humidity ( $>85 \%$ ) environment which is very favorable for sheath blight disease development. This method produces a more precise measure of disease severity (disease index) based upon the disease lesion moving upward along the culm length, similar to criteria followed in field-screening methods (9). Using this microchamber method in the current study, the disease index of the test entries had a wide range, varying from highly susceptible to moderately resistant. The high correlation between the visual score of the test entries and the disease severity measurement signifies that the method is a reliable indicator of sheath blight disease. Similarly, the ranking of the six check cultivars for sheath blight based on field screening $(6,11)$ and the microchamber method validates the accuracy of the microchamber method. The major advantage of this method, compared with the field screening methods, is that a minimum number of seed are required, a very important consideration because the wild Oryza spp. produce few seed. Other advantages of this method are (i) it can be completed within 5 weeks from planting the seed to evaluating the disease reaction, (ii) evaluations can be conducted any time of the year, and (iii) it estimates the physiological response of the individual plants (genotypes) to $R$. solani better than field methods because the effects of the two major confounding factors, plant height and days to heading, are eliminated (11). It is well known that taller plants and those that flower later can easily escape the disease in the field, even with artificial inoculations (23), and most of the wild Oryza spp. accessions fall into at least one of these categories. On the other hand, field screening methods are still needed to evaluate the field resistance of a population under different microenvironments as part of rice cultivar development programs.

The detached-leaf inoculation method separated cvs. Jasmine 85 and TeQing, moderately resistant checks, from Lemont and Cocodrie, highly susceptible checks, based on disease reaction (lesion length). The lesion lengths of the moderately susceptible checks, Bengal and Ahrent, were only slightly different than the highly susceptible checks, Lemont and Cocodrie, suggesting that there is an increased amount of disease pressure on the cut leaf pieces, most likely due to the partial loss of physiological resistance in detached leaf tissue compared with the whole plant used in the microchamber method. Previously,
$\mathrm{Ou}$ (20) reported inoculating detached leaves with sclerotia to screen rice cultivars but sclerotia can produce inconsistent results due to differences in the germination capability of individual sclerotia (Y. Jia, personal communication). In this study, inoculations were done with round mycelial disks collected from the rapidly growing edge of the colony near the edge of the petri dish and similar in age; thus, the $R$. solani was more uniform in virulence because the disks were harvested from approximately the same circumference within the dish (11).

The toothpick method evaluates disease intensity based on the leaf lesion length of both the leaf blade and sheath summed together. Leaf position, another important criterion for sheath blight disease development, also is considered in this disease assessment. Data from the first three leaves were useful in interpreting disease response as reported earlier (6). Also, the correlation between the overall visual rating and lesion length of the primary and secondary tillers was better than the correlation with the ratoon tillers (6). Among the three leaves, lesion length was the longest for the first leaf, which was the oldest leaf. Looseness of leaf sheath around the stem of older leaves compared with younger leaves made the older leaves more susceptible to the disease because it was easier for the mycelia to penetrate into the inner side of the leaf sheath and mechanisms of physiological resistance begin to shut down as the plant ages (20). Using relative lesion length rather than lesion length did not improve the correlation with the visual score. All disease symptoms were measured 7 days after inoculation, which was sufficient time for the sheath blight disease to develop and assess the disease reaction; thus, differences in leaf length were minimized and no differences in lesion length and relative lesion length were identified when these ratings were correlated with the visual ratings. The combined lesion length of the second and third leaves produced the best correlation with the overall visual score $(r$ $=0.78, P<0.01$; Table 2), signifying the best overall picture of the plant's disease response.

The discrepancy between the methods, especially comparing the toothpick method with the other two methods, may be due to the difference in plant architecture as the plant grows and matures. In the toothpick method, adult plants at panicle initiation stage were inoculated whereas, in the microchamber and detached-leaf methods, 4week-old plants were inoculated. The microchamber method appeared to be the best of the three methods assessed in this study for screening the wild Oryza spp. accessions' reaction to the sheath blight disease, for which field screening is not recommended. The toothpick requires a longer period of time, more space, and an envi- 
ronmentally controlled growth chamber which makes it difficult to screen a large number of Oryza spp. accessions or progenies derived from crosses with Oryza spp.

The seven accessions identified as moderately resistant are the best choice for developing germplasm lines with enhanced sheath blight resistance in an adapted background and for genetic mapping studies to identify the chromosomal locations of sheath blight quantitative trait loci (QTL) for eventual fine-mapping of the resistance genes. There are many examples in the literature of incorporating genes, especially for pest resistance, from wild Oryza spp. into cultivated rice as illustrated by the research done at IRRI $(3,10,16)$ and in the United States (19). If the Oryza sp. is genetically more closely related to cultivated rice (O. sativa), such as the ancestral species $O$. nivara and $O$. rufipogon (10), incorporating desirable traits is accomplished more easily than if the desired trait is in an Oryza sp. such as $O$. officinalis which is more distantly related. More recently, the advanced backcross method has been used to develop several mapping populations (18) and, subsequently, release germplasm with improved performance. Mapping populations developed with one of the moderately resistant Oryza spp. accessions as a parent will be used to determine whether novel sheath blight QTL or genes are present in the given Oryza accession. These novel sheath blight genes can then be stacked with known resistance genes to develop rice cultivars with improved sheath blight resistance.

\section{ACKNOWLEDGMENTS}

We thank G. Liu for excellent technical assistance with the microchamber method; Q. Ho and R. Herman for their assistance; and G. Xiang, visiting scientist from the Rice Research Institute, Guizhou Academy of Agricultural Sciences, P. R. China, for part of the data collected using the toothpick method.

\section{LITERAURE CITED}

1. Amante, A. D., de la Pena, R., Sitch, L. A., Leung, H., and Mew, T. W. 1990. Sheath blight $(\mathrm{ShB})$ resistance in wild rices. Int. Rice Res. Newsl. 15:5.

2. Brar, D. S., Elloran, R., and Khush, G. S. 1991.
Interspecific hybrids produced through embryo rescue between cultivated and eight wild species of rice. Rice Genet. Newsl. 8:91-93.

3. Brar, D. S., and Khush, G. S. 1997. Alien introgression in rice. Plant Mol. Biol. 35:35-47.

4. Counce, P. A., Keisling, T. C., and Mitchell, A. J. 2000. A uniform, objective, and adaptive system for expressing rice development. Crop Sci. 40:436-443.

5. Eizenga, G. C., Agrama, H, A., Lee, F. N., and Jia, Y. 2008. Exploring genetic diversity and potential novel disease resistance genes in a collection of rice wild relatives. Genet. Resour. Crop Evol. DOI 10.1007/s10722-008-9345-7

6. Eizenga, G. C., Lee, F. N., and Rutger, J. N. 2002. Screening Oryza species plants for rice sheath blight resistance. Plant Dis. 86:808-812.

7. Hoff, B. J., Rush, M. C., Mcllrath, W. O., and Morgan, A. 1976. 1976 Disease Nurseries, A Preliminary Report. Pages 142-192 in: 68th Annu. Prog. Rep. La. State Univ. Rice Exp. Stn. Crowley, LA.

8. International Rice Research Institute. 1992. Resistance of conserved germplasm to nonspecialized pathogens. Pages 140-143 in: IRRI Program Report for 1992.

9. International Rice Research Institute. 2002. Page 19 in: Standard Evaluation System for Rice (SES). International Rice Research Institute, Manila, Philippines.

10. Jena K. K., and Khush, G. S. 2000. Exploitation of alien species in rice improvementopportunities, achievements and future challenges. Pages 271-285 in: Rice Breeding and Genetics: Research Priorities and Challenges. J. S. Nanda, ed. Science Publishers, Inc., Endfield, $\mathrm{NH}$.

11. Jia, Y., Correa-Victoria, F., McClung, A., Zhu, L., Liu, G., Wamishe, Y., Xie, J., Marchetti, M. A., Pinson, S. R. M., Rutger, J. N., and Correll, J. C. 2007. Rapid determination of rice cultivar response to the sheath blight pathogen Rhizoctonia solani using a micro-chamber screening method. Plant Dis. 91:485-489.

12. Jia, Y., Singh, P., Eizenga, G. C., Lee, F. N., and Cartwright, R. D. 2002. In vitro identification of cultivar responses to rice sheath blight pathogen Rhizoctonia solani. Pages 229-236 in: B. R. Wells Rice Research Studies. R. J. Norman and J. F. Meullenet, eds. Univ. Arkansas Agric. Exp. Stn. Ser. 504 Fayetteville, AR.

13. Khush, G. S., Balacangco, E., and Ogawa, T. 1990. A new gene for resistance to bacterial blight from Oryza longistaminata. Rice Genet. Newsl. 7:121-122.

14. Khush, G. S., Ling, K. C., Aquino, R. C., and Aquiero, V. M. 1977. Breeding for resistance to grassy stunt in rice. Pages 3-9 in: Proc. 3rd Int. Congr. SABRAO Plant Breed. Pap. 1(4). Canberra, Australia.

15. Kovach, W. L. 2005. MVSP-A Multivariate
Statistical Package for Windows, ver. 3.1. Kovach Computing Services, Pentraeth, Wales, U.K.

16. Lakshmanan, P. 1991. Resistance to sheath blight (ShB) and brown spot (BS) in lines derived from Oryza officinalis. Int. Rice Res. Newsl. 16:8.

17. Lee, F. N., and Rush, M. C. 1983. Rice sheath blight: a major rice disease. Plant Dis. 67:829832.

18. McCouch, S. R., Sweeney, M., Li, J., Jiang, H., Thomson, M., Septiningsih, E., Edwards, J., Moncada, P., Xiao, J., Garris, A., Tai, T., Martinez, C., Tohme, J., Sugiono, M. McClung, A., Yuan, L. P., and Ahn, S. N. 2007. Through the genetic bottleneck: $O$. rufipogon as a source of trait-enhancing alleles for $O$. $s a$ tiva. Euphytica 154:317-339.

19. Oster, J. J. 1992. Reaction of a resistant breeding line and susceptible California rice cultivars to Sclerotium oryzae. Plant Dis. 76:740744.

20. Ou, S. H. 1985. Pages 256-268 in: Rice Diseases. Commonwealth Mycological Institute, Kew, England.

21. Pan, X. B., Rush, M. C., Sha, X. Y., Linscombe, S. D., Stetina, S. R., and Oard, J. 1999. Major gene, nonallelic sheath blight resistance from the rice varieties Jasmine 85 and TeQing. Crop Sci. 39:338-346.

22. Park, D.-S., Sayler, R. J., Hong, Y.-G., Nam, M.-H., and Yang, Y. 2008. A method for inoculation and evaluation of rice sheath blight disease. Plant Dis. 92:25-29.

23. Pinson, S. R. M., Capdevielle, F. M., and Oard, J. H. 2005. New and confirmed QTLs conditioning sheath blight resistance in rice using recombinant inbred lines. Crop Sci. 45:503510.

24. Rush, M. C., and Lee, F. N. 1992. Sheath blight. Pages 22-23 in: Compendium of Rice Diseases. R. K. Webster and P. S. Gunnell, eds. American Phytopathological Society, St. Paul, MN.

25. Savary, S., and Mew, T. W. 1996. Analyzing crop losses due to Rhizoctonia solani: Rice sheath blight, a case study. Pages 237-245 in: Rhizoctonia Species: Taxonomy, Molecular Biology, Ecology, Pathology and Disease Control. B. Sneh, S. Jabajihare, S. Neate, and G Dijst, eds. Kluwer Academic Publisher, Dordrecht, The Netherlands.

26. Singh, A., Rohilla, R., Singh, U. S., Savary, S. Willocquet, L., and Duveiller, E. 2002. An improved inoculation technique for sheath blight of rice caused by Rhizoctonia solani. Can. J. Plant Pathol. 24:65-68.

27. Wamishe, Y. A., Jia, Y., Singh, P., and Cartwright, R. D. 2007. Identification of field isolates of Rhizoctonia solani to detect quantitative resistance in rice under greenhouse conditions. Front. Agric. China 1:361-367. 